\title{
The role of structural flexibility in plasmon-driven coupling reactions: kinetic limitations in the dimerization of nitro-benzenes
}

Authors: W. Koopman ${ }^{a)}$, E. Titov $^{b)}$, R.M. Sarhan ${ }^{a), e)}$, T. Gaebel ${ }^{b)}$, R. Schürmann ${ }^{b}$, A. Mostafa ${ }^{b)}$, S. Kogikoski Jr. ${ }^{b)}$, A.R. Milosavljevic ${ }^{d)}$, F. Stete ${ }^{a)}$, F. Liebig ${ }^{b)}$, C.N.Z. Schmitt ${ }^{c}$, J. Koetz ${ }^{b}$, I. Bald ${ }^{b)}$, P. Saalfrank ${ }^{b)}$, M. Bargheer ${ }^{a), e)}$

a) University of Potsdam, Institute of Physics and Astronomy, 14476 Potsdam, Germany

b) University of Potsdam, Institute for Chemistry, 14476 Potsdam, Germany.

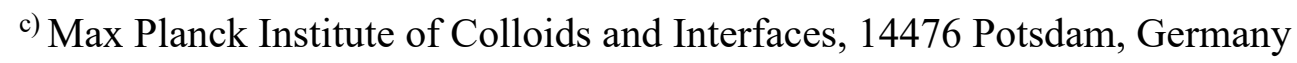

d) Synchrotron SOLEIL, 91192 GIF-sur-YVETTE CEDEX, France

e) Helmholtz Zentrum Berlin für Materialien und Energie, Albert-Einstein-Str. 15, 12489 Berlin, Germany

E-mail: bargheer@uni-potsdam.de

\begin{abstract}
The plasmon-driven dimerization of 4-nitrothiophenol (4NTP) to 4-4'-dimercaptoazobenzene

(DMAB) has become a testbed for understanding bimolecular photoreactions enhanced by nanoscale metals, in particular, regarding the relevance of electron transfer and heat transfer from the metal to the molecule. By adding a methylene group between the thiol bond and the nitrophenyl, we add structural flexibility to the reactant molecule. Time-resolved surface-enhanced Raman-spectroscopy proves that this (4-nitrobenzyl)mercaptan (4NBM) molecule has a larger dimerization rate and dimerization yield than 4NTP and higher selectivity towards dimerization. X-ray photoelectron spectroscopy and density functional theory calculations show that the electron transfer would prefer activation of 4NTP over 4NBM. We conclude that the rate limiting step of this plasmonic reaction is the dimerization step, which is dramatically enhanced by the additional flexibility of the reactant. This study may serve as an example for using nanoscale metals to simultaneously provide charge carriers
\end{abstract}


for bond activation and localized heat for driving bimolecular reaction steps. The molecular structure of reactants can be tuned to control the reaction kinetics.

\section{Introduction}

Photosynthesis requires the light-induced formation of chemical bonds. The emerging field of heterogeneous photocatalysis via plasmon excitations, in short plasmon-chemistry, has shown great potential as platform for efficient light-driven bond formation. In plasmon-driven chemistry, noblemetal nanostructures act as light-antennas and convert photon energy to energetic charges and localized heat ${ }^{1-4}$. On the one hand, the high charge densities in metal nanoparticles allow the activation of several molecules simultaneously, which facilitates coupling reactions of two or more molecules ${ }^{5-7}$. On the other hand, nanoscale heating can increase the speed of molecular reorganization and surface diffusion, thereby increasing the rate of favorable reactive encounters. Several important examples of plasmondriven bond formation were demonstrated in recent years ${ }^{8,9}$, including $\mathrm{C}-\mathrm{C}$ bond formation in hydrocarbons ${ }^{5,10}$, Suzuki-Miyaura type reactions ${ }^{11}$ and molecular dimerization reactions by $\mathrm{N}=\mathrm{N}$ bond formation $^{5,12,13}$.

Until recently, the question if the dominating effect of plasmonic nanoparticles is to act as charge donors $^{14,15}$ or heat sources ${ }^{12,16}$ has been heavily debated. Nowadays, most authors agree that depending on the reaction, one or the other effect can dominate ${ }^{17}$. For example, the dissociation of diatomic compounds on gold-particles, in particular of $\mathrm{H}_{2}{ }^{1,18}$ and $\mathrm{O}_{2}{ }^{19}$, is likely determined by the transfer of energetic electrons, while the fragmentation of organic molecules, such as the decomposition of dicumyl-peroxide ${ }^{16}$, is rather dominated by the plasmon-mediated photo-heating. Although much progress has been made in understanding such dissociation reactions, our understanding of plasmondriven multistep bond-formation processes is still in its infancy. In these reactions, different reaction steps might profit from the presence of plasmonic excitations. As a prominent example, the dimerization reaction of 4-nitrothiophenol (4NTP) to 4-4'-dimercaptoazobenzene (DMAB) was confirmed by many 
authors to occur only in the presence of light ${ }^{12,20-22}$. Hence the role of electrons was emphasized. On the other hand, we recently demonstrated that the rate of this reaction is determined by the plasmon-induced temperature increase of the particles ${ }^{7}$. We reconciled these seemingly contradictory observations by speculating that the dimerization progresses is a tandem reaction ${ }^{7}$, which features two reaction barriers: for the initial activation of the reactants and for their subsequent dimerization. The first barrier may be overcome by providing energetic electrons to the reactants while the second barrier seems to be overcome by the local photo-induced temperature-rise ${ }^{7}$. In such a tandem-reaction mechanism, energy dissipated from the electron- to the phonon-system is not lost. In contrast, the released vibrational heat is a necessary ingredient to allow the reaction to proceed.

Recently another possible mechanism that could explain the temperature dependence has come to attention $^{4}$ : If the particles act as redox centers, an imbalance in the oxidation and reduction halfreactions can cause a charging of the particles, which is equivalent to a shift in the chemical potential $\mu$. This shift and the broadening of the Fermi-distribution together can be sufficiently large for electrons to thermally overcome the injection barrier. In an orbital picture, sometimes this process is described by hopping of an electron into the LUMO at $\mathrm{E}_{\mathrm{LUMO}}$ of the adsorbate, although the extra electron definitely modifies the orbital energies. In any case, if the activation energy $E_{a}=E_{L U M O}-\mu \gg k_{B} T$ the Fermi-distribution can be approximated by the Boltzmann distribution, and Arrhenius-like temperature dependence of the reaction rate $\left(k \propto e^{-E_{a} / k_{B} T_{e}}\right)$ would then result because of the electronic temperature $T_{e}$. If $T_{e}$ was the relevant factor, energy dissipated from the electron- to the phonon-system would be lost. For the design of efficient nanoscale metal catalysts the distinction of electronic and vibrational temperatures in nonequilibrium is of high importance, because - as we will show - the heat dissipated to vibrations is not lost at all: It facilitates the dimerization step.

In this article, we compare the well-studied plasmon-induced dimerization of 4NTP to DMAB to the dimerization of (4-nitrobenzyl)mercaptan (4NBM) to 4-,4'-dimercaptomethan-azobenzene (DMMA). In 4 NBM the benzene ring is separated from the thiol bond to the gold surface by an additional methylene group compared to 4NTP. This increases its thermal motion due to the additional structural 
flexibility and hence the volume accessible to the reactive nitro group of NBM for finding its reaction partner as schematically sketched in Figure 1. The motional freedom is confirmed by density functional theory (DFT) calculations. On the other hand, the additional methylene group decreases the probability of transferring an electron to the molecule and in particular to the functional nitro group. DFT calculations find the electron affinity of 4NTP larger than for 4NBM and X-ray photoelectron spectroscopy (XPS) confirms that for Au nanoparticles decorated with 4NTP the reduced work function is $0.6 \mathrm{eV}$ lower than for particles covered by $4 \mathrm{NBM}$. Although any electron driven activation of the nitro-group is more likely for 4NTP, our time resolved surface-enhanced Raman data on an Aunanotriangle template clearly show that the formation of DMMA not only progresses faster than the formation of $\mathrm{DMAB}$, but it also has a higher reaction yield. This confirms that the rate limiting step in the plasmon-driven dimerization of nitro-benzo-molecules to form azo-compounds is the temperature driven structural molecular motion including rotation, vibration and libration which brings the reactive molecular groups close enough for the $\mathrm{N}=\mathrm{N}$ bond to form. The supply of energetic electrons is necessary but not rate limiting.

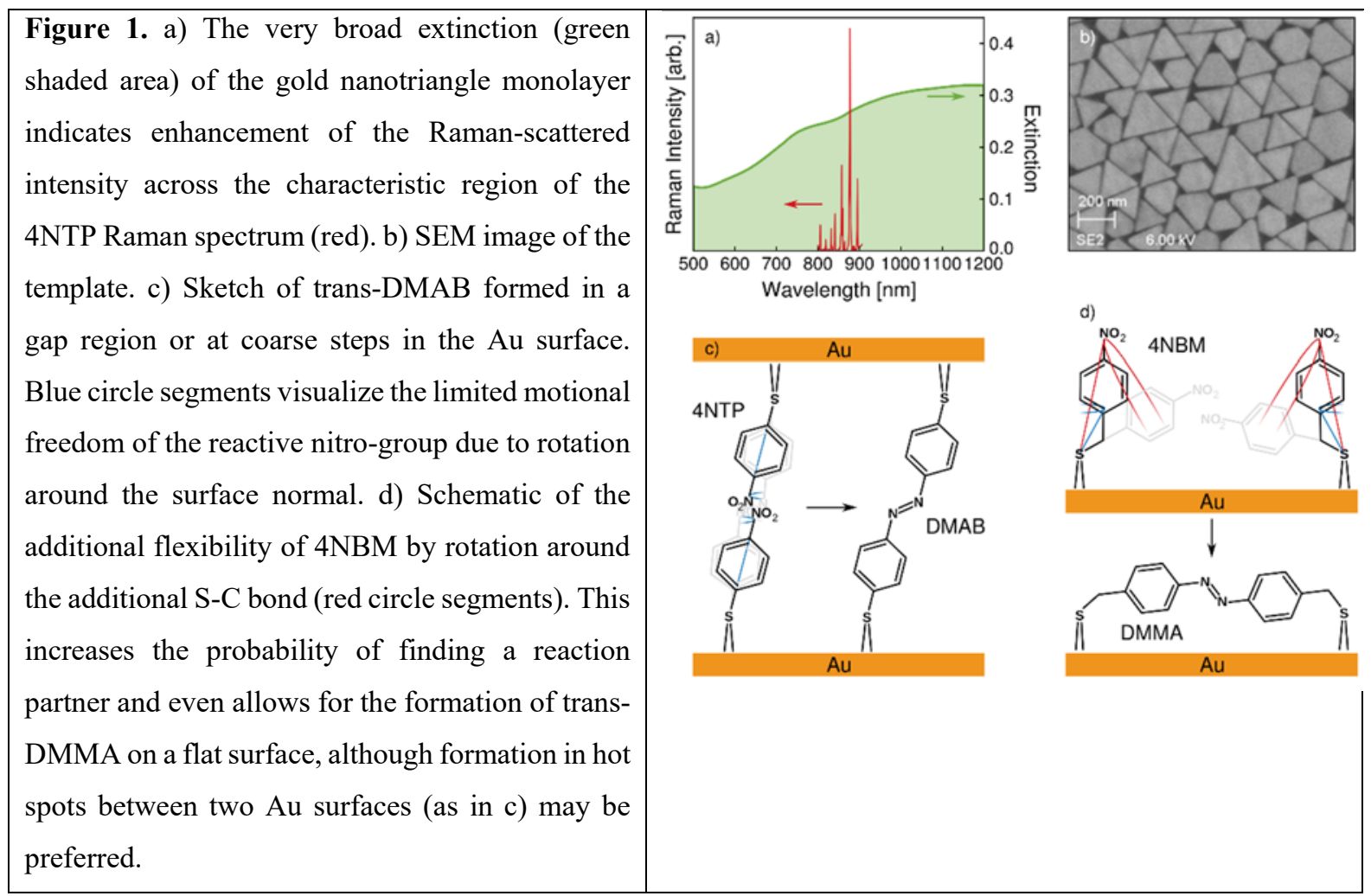




\section{Charge injection into 4NTP and 4NBM on Au-particle surfaces}

Before we discuss the light driven dimerization of 4NTP and 4NBM on Au nanotriangles, we present experimental evidence and arguments from density functional theory (DFT) calculations which show that electron transfer to 4NTP is preferred over electron transfer to 4NBM.

We use X-ray photoelectron spectroscopy (XPS) to characterize the electronic density of states of a Aunanoparticle (AuNP)/ligand system consisting of spherical Au particles $(10 \mathrm{~nm})$ decorated by 4NTP and 4 NBM that are injected as a particle beam into the ultrahigh vacuum. In the continuous beam of AuNPs, X-ray induced damage of the ligand molecules is irrelevant. Since the Fermi-level of the free AuNPs cannot be referenced to the Fermi-energy of the detector system and thus calibrated according to standard binding energy values, we reference binding energies of the functionalized nanoparticle measured at different photon energies $E_{P h}$ to the electronic states of the carrier Ar gas (see methods). The kinetic energy of the photoelectrons generated in the NPs is given by:

$$
\left.E_{\text {kin }}^{A u}=E_{P h}-E_{B}-\Delta_{r e f}^{N T P / N B M} \quad \text { (eq. } 1\right)
$$

where $E_{B}$ is the binding energy of electrons with respect to the AuNP Fermi level and $\Delta_{r e f}^{N T P / N B M}$ is the reduced work function of the system. It is defined as the difference between Fermi level of the AuNPs and the vacuum energy of the Ar reference and depends on the surface dipole of the respective $\mathrm{NP} /$ ligand system. The left side of Figure 2 shows the density of states as a function of $E_{B}$ measured at the photon energy $E_{P h}=100 \mathrm{eV}$ for the conduction band of $\mathrm{Au}$ and the ligand valence orbitals and $E_{P h}=400 \mathrm{eV}$ for the $\mathrm{Au} 4 \mathrm{f}$ levels. In both cases $E_{B}$ is corrected by the reduced work function $\Delta_{r e f}^{N T P / N B M}$ determined from the experimentally measured $\mathrm{Au} 4 \mathrm{f}_{7 / 2}$ energy with respect to the vacuum level reference and the $\mathrm{Au} 4 \mathrm{f}_{7 / 2}$ binding energy of $84.0 \mathrm{eV}$ for metallic gold with respect to the Fermi level. ${ }^{23} \Delta_{r e f}^{N T P}=\Delta_{r e f}^{N B M}-0.6 \mathrm{eV}$ indicates that the corrected work function for the system with 4NTP ligands is $0.6 \mathrm{eV} \pm 0.2 \mathrm{eV}$ smaller than for the system with $4 \mathrm{NBM}$. 
The schematic on the right of Figure 2 clarifies the energetics. The central finding here is that the structureless continua of the electronic density of states near the Fermi level relevant for plasmon mediated reactions are essentially identical for the two systems. The inelastic mean free path of the generated photoelectrons is below $1 \mathrm{~nm}$, hence mainly the gold atoms at the interface and the ligands are probed. ${ }^{24}$ In this system the electron density of the conduction band is strongly altered due to the thiol bond resulting in a rather structureless spectrum. ${ }^{25}$

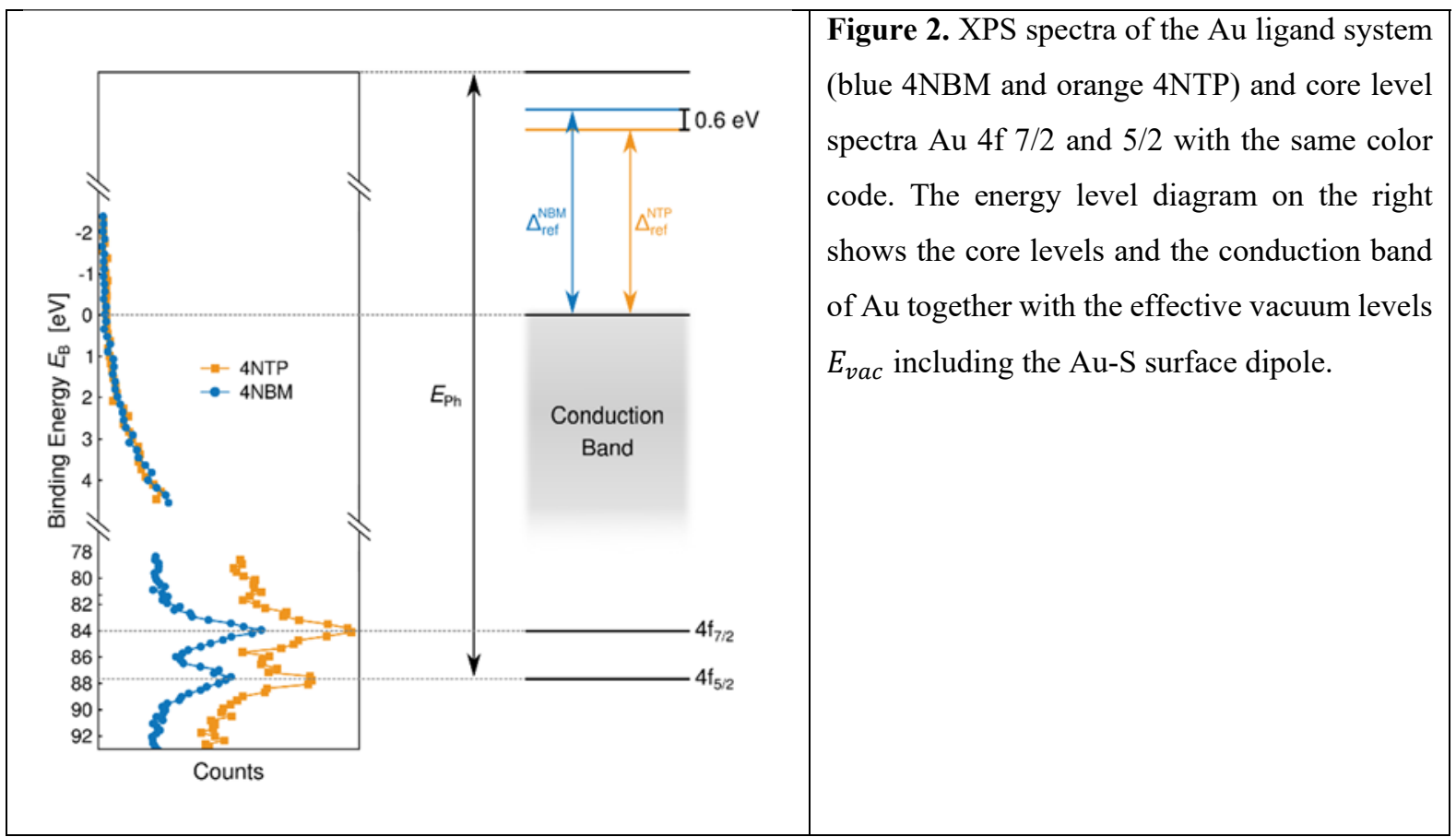

The work-function at the metal-organic interface depends on the surface dipoles formed due to the hybridized metal-molecule states. ${ }^{26-28}$ Although the work functions remain unknown, we find that the reduced work function $\Delta_{r e f}^{N T P}$ of $4 \mathrm{NTP}$ is lower than $\Delta_{r e f}^{N B M}$ for $4 \mathrm{NBM}$, which hints at a higher electron transfer probability from the Au to the molecular system. ${ }^{29}$

The fact that electrons are more readily transferred to the 4NTP system is also corroborated by density functional theory calculations which predict a higher electron affinity for 4NTP. Specifically, using the B3LYP hybrid functional (see Methods section), the adiabatic electron affinities of free 4NTP and free 4NBM calculated by the Delta SCF method are both $1.28 \mathrm{eV}$. Replacing the thiol $\mathrm{H}$ by a single Au atom 
and reoptimizing, gives adiabatic electron affinities of $\mathrm{EA}_{\mathrm{ad}}(\mathrm{Au}-\mathrm{NTP})=2.45 \mathrm{eV}$ and $\mathrm{EA}_{\mathrm{ad}}(\mathrm{Au}-$ $\mathrm{NBM})=2.17 \mathrm{eV}$, i.e., a larger electron affinity for the molecule-"surface" complex. Using gold clusters of increasing size reduces this difference, but it does not change the qualitative behavior (see Supporting Information (SI) in section S2). This is expected to facilitate an electron-mediated photoreaction of 4NTP compared to 4NBM.

\section{NTP and NBM assembled on Au-nanotriangles}

We investigated the reactivity of $4 \mathrm{NTP}$ and $4 \mathrm{NBM}$ on a previously described, ${ }^{30}$ self-assembled template for surface enhance Raman scattering (SERS) consisting of gold nanotriangles (Figure 1b). Besides a good Raman enhancement across a broad spectral range of the plasmon resonance (Figure 1a), this template has demonstrated the ability to support plasmon-driven dimerization of $4 \mathrm{NTP}^{13}$.

4NBM is structurally comparable to $4 \mathrm{NTP}$, but more flexible, due to the additional methylene group between the S-Au bond and the aromatic system (Figure 1c,d and Figure 4). In the SERS spectra shown in Figure 3, this structural similarity is reflected in the similarity of the three dominant modes around $1083 \mathrm{~cm}^{-1}, 1322 \mathrm{~cm}^{-1}$, and $1569 \mathrm{~cm}^{-1}$ for 4NTP (orange) and slightly shifted bands at $1107 \mathrm{~cm}^{-1}, 1344$ $\mathrm{cm}^{-1}$ and $1594 \mathrm{~cm}^{-1}$ for 4NBM (blue). 

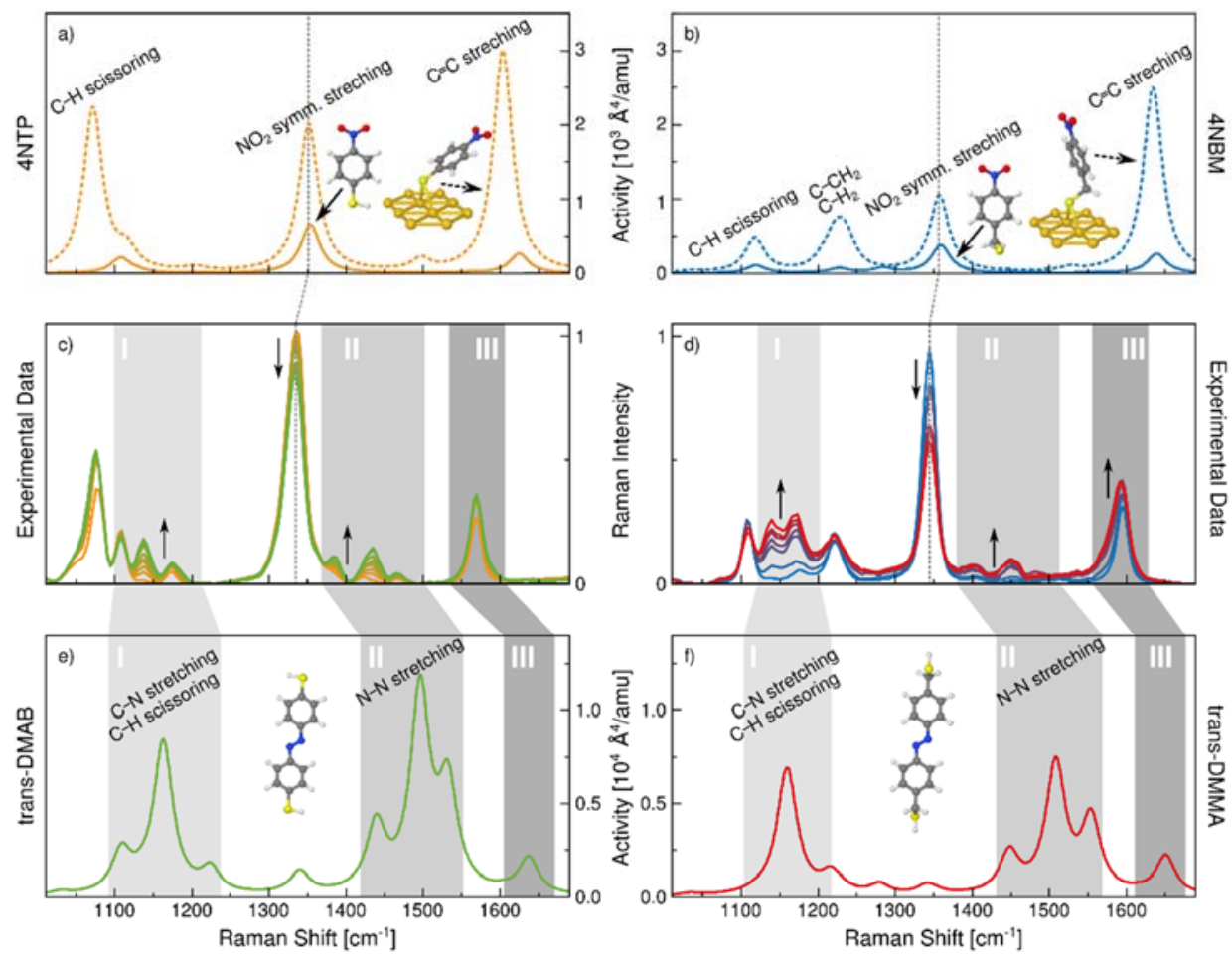

Figure 3. Comparison of experimental SERS spectra and simulated spectra. DFT (B3LYP/TZVP) modeling of a) 4NTP (orange) and b) 4NBM (blue). Solid lines indicate modeling of free molecules. Dashed lines represent spectra of the molecules bound to the Au surface as indicated in the inset. The enhanced Raman polarizability of the complexes (dashed) reproduces the dominant modes of the reactants. The dimerization upon prolonged illumination of the samples by $785 \mathrm{~nm}$ laser light at $15 \mathrm{~mW}$ causes spectral changes in three regions of the experimental spectra which are color coded for the dimerization of c) 4NTP to DMAB (orange to green) d) 4NBM to DMMA (blue to red). DFT spectra of the products (free molecules) are plotted in e) DMAB (green) and f) DMMA (red). The azo-products are identified by the main features of trans-DMAB (e) and trans-DMMA (d) attributed to $\mathrm{C}-\mathrm{N}$ stretching and $\mathrm{C}-\mathrm{H}$ scissoring (region I) and $\mathrm{N}=\mathrm{N}$ vibrational modes (region II) by the DFT calculations. The consumption of the product is concluded from the loss of intensity at the $\mathrm{NO}_{2}$ vibration. No frequency scaling is applied to the simulations, which are broadened by Lorentzians with a width of $15 \mathrm{~cm}^{-1}$.

The upper row of Figure 3 depicts DFT (B3LYP/TZVP) calculations of Raman spectra of the isolated reactant molecules 4NTP (orange) and 4NBM (blue), which already agree well with the main features of the experimental spectra in the middle row with the same color code. The experimental peaks can be assigned to modes with predominant $\mathrm{C}-\mathrm{H}$ symmetric bending $\left(1083 \mathrm{~cm}^{-1} / 1107 \mathrm{~cm}^{-1}\right), \mathrm{NO}_{2}$ symmetric stretching $\left(1322 \mathrm{~cm}^{-1} / 1344 \mathrm{~cm}^{-1}\right)$ and $\mathrm{C}=\mathrm{C}$ stretching $\left(1569 \mathrm{~cm}^{-1} / 1594 \mathrm{~cm}^{-1}\right)$ character, for (4NTP/4NBM) respectively (see SI for mode assignments in Table S2). From DFT calculations in which the 4NTP and 4NBM molecules were adsorbed on gold clusters of various size, we find that both 
molecules adsorb preferentially at a bridge position and are tilted with respect to the surface normal (see insets in Figures $3 \mathrm{a}$ and $\mathrm{b}$ for the case of $10 \mathrm{Au}$ atom clusters to mimic an $\mathrm{Au}(111)$ surface). This is consistent with earlier investigations which presented evidence (for a self-assembled monolayer structure) for an adsorption of 4NTP on gold via a covalent S-Au bond, with a tilt of the ring away from the surface normal ${ }^{31}$.

The additional methylene group in 4NBM on the other hand allows an upright arrangement of the ring ${ }^{32-}$ ${ }^{34}$ as can be seen in Figure $3 \mathrm{~b}$. The extra $\mathrm{CH}_{2}$ group allows for additional flexibility due to rotation around the additional S-C bond. Figure 4 shows DFT modeling of this hypothesis. The blue curves depict the energy required to rotate the 4-NBM molecule around the surface normal (solid line) and around the S-C bond (dashed line) as indicated in the color coded insets. The orange line shows that also for 4NTP a rotation by about $20^{\circ}$ around the surface normal is feasible at room temperature (thermal energy of $25 \mathrm{meV}$ ). However, the additional $\pm 40^{\circ}$ rotation of $4 \mathrm{NBM}$ around the $\mathrm{S}-\mathrm{CH}_{2}$ bond dramatically increases the flexibility and hence the volume accessible to the reactive nitro group. (Further cluster models and adsorption structures, also for charged species, can be found in Sec. S2 of the SI.)

The additional flexibility is also reflected in the Raman feature at $1221 \mathrm{~cm}^{-1}$ for the adsorbed 4NBM molecules which can be assigned to the $\mathrm{C}($ phenyl)- $\mathrm{C}(\mathrm{H} 2)$ stretching and $\mathrm{H}$ wagging mode involving the methylene group plus $\mathrm{C}-\mathrm{H}$ scissoring at the phenyl ring. Moreover, the Au surface modifies the calculated Raman spectra for the adsorbed 4NTP and 4NBM molecules as follows: (i) The underlying gold surface leads to a considerable enhancement of Raman intensities as expected from the increased polarizability. Field-enhancement effects are not included in the calculation. (ii) New features appear which bring theory and experiment in even better agreement: For instance, for 4NTP new low-intensity features are seen around $1200 \mathrm{~cm}^{-1}$, as in the experiment. (iii) Some of the free-molecule peaks shift upon adsorption, in particular for 4NTP, which is less decoupled from the surface. For 4NTP, notably the low- and high-energy peaks around $1100 \mathrm{~cm}^{-1}$ and $1600 \mathrm{~cm}^{-1}$ shift to the red, while the feature related to $\mathrm{NO}_{2}$, pointing away from the surface, is largely unaffected. The larger distance to the $\mathrm{Au}$ 
surface also explains, why the $\mathrm{NO}_{2}$ peak in the simulation of adsorbed molecules has a low relative intensity compared to the other modes. The intensity in the experiments is dominated by local conformations of the nanoscale metal template, which produce hot spots between two metal surfaces.

\begin{tabular}{|l|l|l|}
\hline inTP (orange squares) and 4NBM around the \\
modeling of the energy required for the rotation of \\
surface normal (blue circles) for molecules \\
assuming the relaxed equilibrium in the bridge- \\
adsorption. Energy for the additional rotation of \\
4NBM around the $\mathrm{S}-\mathrm{CH}_{2}$ bond is shown as blue \\
pentagons. At room temperature (25 meV) the \\
combination of both rotations makes a large \\
volume accessible to the reactive nitro group, and \\
in particular it allows for the formation of dimers \\
on the same Au surface.
\end{tabular}

\section{Plasmon-driven dimerization of nitro-aromatics}

Prolonged illumination of the sample by the $785 \mathrm{~nm}$ Raman-laser at $15 \mathrm{~mW}$, leads to the gradual appearance of new features in the 4NTP and 4NBM spectra (Figure 3c,d). It is well established that in the case of 4 NTP these peaks indicate the formation of $\mathrm{DMAB}^{9}$. Due to the structural similarity of 4NTP and 4NBM, we presume that the newly appearing features in the 4NBM spectrum originate from the dimerization product DMMA. This claim is supported by the DFT modeling of the Raman spectra of both dimers. Indeed, the simulated spectra of isolated trans-DMAB (Figure 3e, green) and transDMMA (Figure 3f, red) molecules show maxima in the regions where the experimental spectra exhibit considerable intensity increase, even without the addition of the Au surfaces. The DFT modeling of cisspectra of both products shows considerably less agreement with experiment (see SI Figure S5).

The main resonances in the simulated spectra of the products can be readily assigned to the experimentally appearing features, between $1075 \mathrm{~cm}^{-1}$ and $1200 \mathrm{~cm}^{-1}$ (region I) and $1400 \mathrm{~cm}^{-1}$ and 1540 
$\mathrm{cm}^{-1}$ (region II). A shift in wavenumbers between experiment and simulation is likely caused by interaction of the molecules with the particle surface. In accordance with literature ${ }^{35}$, our DFT calculations of DMAB assigns the experimental peaks at 1134,1387 , and $1434 \mathrm{~cm}^{-1}$ to modes of combined $\mathrm{C}-\mathrm{H}$ scissoring, $\mathrm{C}-\mathrm{N}$ symmetric stretching and $\mathrm{N}=\mathrm{N}$ stretching vibrations, respectively (Figure 3e). Similarly, we assign the high energy resonances in the 4NBM spectrum (region II) to the DMMA-modes dominated by $\mathrm{N}=\mathrm{N}$ stretching and the low-energy resonances (region I) to the modes dominated by the C-H scissoring and C-N stretching (Figure 3f). In the experiments two resonances increase in the latter region. The DFT simulation of isolated DMMA predicts two modes with very small spacing, which might increase upon adsorption (See SI Figure S8n for DFT spectra without broadening).

For both reactions, the simulated Raman scattering cross-sections of the dimer form are much higher than those of the monomer form (compare Figures 3a,b to 3e,f). Indeed, the cross-section of the highest C-N stretching vibration of DMAB (DMMA) is about a factor of about 20 higher than the $\mathrm{NO}_{2}$ stretching vibration of the isolated 4NTP (4NBM). From the low intensity of the dimer peaks in the experimental Raman spectra thus it follows that only a small fraction of the reactants converts to dimers. This is consistent with the small reduction of the experimental intensity at the $\mathrm{NO}_{2}$ peak.

\section{Dimerization kinetics}

The product generation kinetics for both reactions can best be studied using the transients of the product peaks in region I. In this region, DMAB shows a combined $\mathrm{C}-\mathrm{N}$ stretching and $\mathrm{C}-\mathrm{H}$ bending resonance at $1137 \mathrm{~cm}^{-1}$, while 4 NTP does not have any resonance at the same wavenumber. Thus, the intensity $I_{D M A B}$ of the DMAB peak should exclusively correspond to the generation of azo-dimer. The situation is somewhat more difficult for the 4NBM dimerization, as the reason for the apparent double peak of the DMMA product in region I is not known. Moreover, 4NBM has a methylene related resonance at $1176 \mathrm{~cm}^{-1}$ which overlaps with the product peak at $1169 \mathrm{~cm}^{-1}$. To prevent crosstalk between the reactant 
and product kinetics we chose the resonance at $1140 \mathrm{~cm}^{-1}$ to represent the product formation kinetics via $I_{D M M A}$. Interestingly, the kinetics at $1169 \mathrm{~cm}^{-1}$ are nearly identical (See SI Figure S9).

Figure 5 shows a comparison of the formation kinetics for both dimers. It strikes immediately that the non-normalized kinetics show a much higher intensity for the 4NBM/DMMA dimerization than for the case of 4NTP/DMAB. While experimental Raman scattering intensities from different molecules cannot be generally compared quantitatively, the DFT calculations (Figure 3e,f) show that the Raman cross-section of the $\mathrm{N}=\mathrm{N}$ resonance of DMAB is slightly higher than of DMMA. The higher DMMA signal must be caused by a higher dimerization yield for the 4NBM/DMMA reaction.

To determine the difference more quantitatively, we extracted the rate by fitting a kinetic model. The formation rate of DMAB is limited by the bi-molecular dimerization step ${ }^{7,36,37}$. We expect this to be the case for the formation of DMMA as well. This suggests the following kinetics model for the Raman intensity of the formed dimer product (See SI section S5 for derivation):

$$
I_{p}(t)=I_{p}^{\infty} \cdot \frac{k_{d i m} t}{1+k_{d i m} t}
$$

Here, the rate constant $k_{d i m}=2\left[R_{0}\right] k_{2}$ of the dimerization includes the initial concentration $\left[R_{0}\right]$ of the reactant and $k_{2}$ is the second order rate constant. The Raman scattering intensity $I_{p}^{\infty}$ in the limit $t \rightarrow$ $\infty$ is a fitting parameter ${ }^{7}$. The dimerization reaction rate constants $k_{\text {dim }}^{D M A}=0.14 \pm 0.031 / \mathrm{s}$ obtained from the time resolved Raman data in Figure 5 for the formation of DMMA is 3.5 times higher than for $\mathrm{DMAB}, k_{\operatorname{dim}}^{D M A B}=0.04 \pm 0.0081 / \mathrm{s}$. Both fits agree well with the data. The significantly higher formation rate for DMMA compared to $\mathrm{DMAB}$, clearly suggest that the reaction is limited by the activation barrier for the structural rearrangement. 


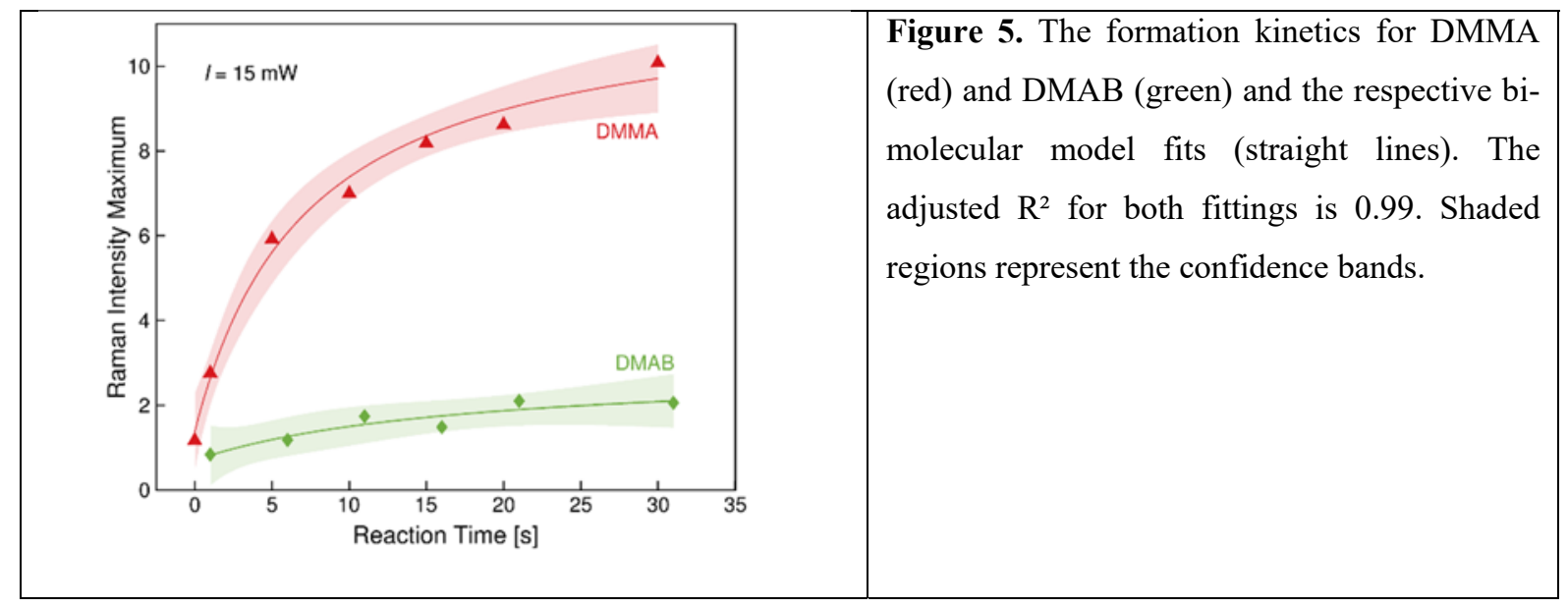

\section{Selectivity of the plasmon driven dimerization}

It is well known that not all activated 4NTP molecules dimerize to form DMAB, because only a fraction of molecules has the right geometric arrangement. Instead, a considerable fraction of molecules converts to single thiophenol compounds such as amino-thiophenol (4ATP) ${ }^{38}$ or un-functionalized thiophenol (TP) ${ }^{39}$. Dissociative electron attachment was also observed in gas phase experiments. ${ }^{40}$

Determining the yield of a reaction from Raman spectra is generally difficult, as the precise Raman scattering cross-section of a single molecule is usually not known. The situation is even more complicated in SERS measurements due to very inhomogeneous amplification of the Raman signal in plasmonic hot spots. However, we can use the information obtained from the kinetics measurements to determine the selectivity for the dimerization process. We fitted the intensity change of the reactant bands $\Delta I^{N O_{2}}(t)=I_{0, R}^{N_{2}}-I^{N O_{2}}(t)$ from Figure $3 \mathrm{c}, \mathrm{d}$ with

$$
\Delta I^{N O_{2}}(t)=I_{d i s}^{N O_{2}}\left(1-e^{-k_{1} t}\right)+I_{d i m}^{N O_{2}}\left(1-\frac{1}{1+k_{d i m} t}\right)=\Delta I_{d i s}^{N O_{2}}(t)+\Delta I_{d i m}^{N O_{2}}(t),
$$

(eq.3)

where $\mathrm{I}_{0, R}^{\mathrm{NO}_{2}}$ corresponds to the intensity of the initial $\mathrm{NO}_{2}$ band and $I^{\mathrm{NO}_{2}}(t)$ is the intensity of this band after a time $t$. (See SI S5 for derivation.) $k_{\text {dim }}^{D M M A}$ and $k_{\text {dim }}^{D M A B}$ are the respective rate constants, obtained by fitting the product formation kinetics, as discussed in the previous section. Eq. 3 assumes in addition 
to the dimerization channel (quantified via $\mathrm{Idim}^{\mathrm{NO}_{2}}$ ) the loss of reactants via dissociation, i.e., a unimolecular reaction described by an exponential decay with rate constant $k_{1}$, which is quantified by the intensity contribution $\mathrm{I}_{d i s} \mathrm{NO}_{2}$ to the $\mathrm{NO}_{2}$ band. The orange squares in Figure 6a and blue circles in Figure $6 \mathrm{~b}$ represent the total change of reactant relative to the maximum dimerization contribution $\Delta I^{\mathrm{NO}_{2}}(t) / I_{d i m} \mathrm{NO}_{2}$. By virtue of eq. 3 this signal of the decaying reactant molecules identified by the $\mathrm{NO}_{2}$ vibration can be written as a sum of two contributions, where the second is identical to the measure of the product formation $I_{p}\left(t_{i}\right) / I_{p}^{\infty}$, which is represented by the green diamonds in Figure 6a and red triangles in Figure 6b:

$$
\frac{\Delta I^{N O_{2}}(t)}{I_{d i m}^{N O_{2}}}=A\left(1-e^{-k_{1} t}\right)+\frac{k_{\operatorname{dim}} t}{1+k_{\operatorname{dim}} t}
$$

For the 4NBM molecule the fitting parameter $\mathrm{A}=I_{\text {dis }}^{\mathrm{NO}_{2}} / \mathrm{I}_{\mathrm{dim}}^{\mathrm{NO}_{2}}$ is zero, i.e. essentially all molecules dimerize, whereas for the 4NTP product a large fraction of the reactant molecules are decomposed without forming dimers. In Figure 6 the first and second term are plotted as dashed and dotted lines, respectively, and the second one agrees with product formation $I_{p}(t) / I_{p}^{\infty}$. In this way we can identify the kinetics of the product formation and the reactant consumption within our model without having to quantify the Raman cross sections. 


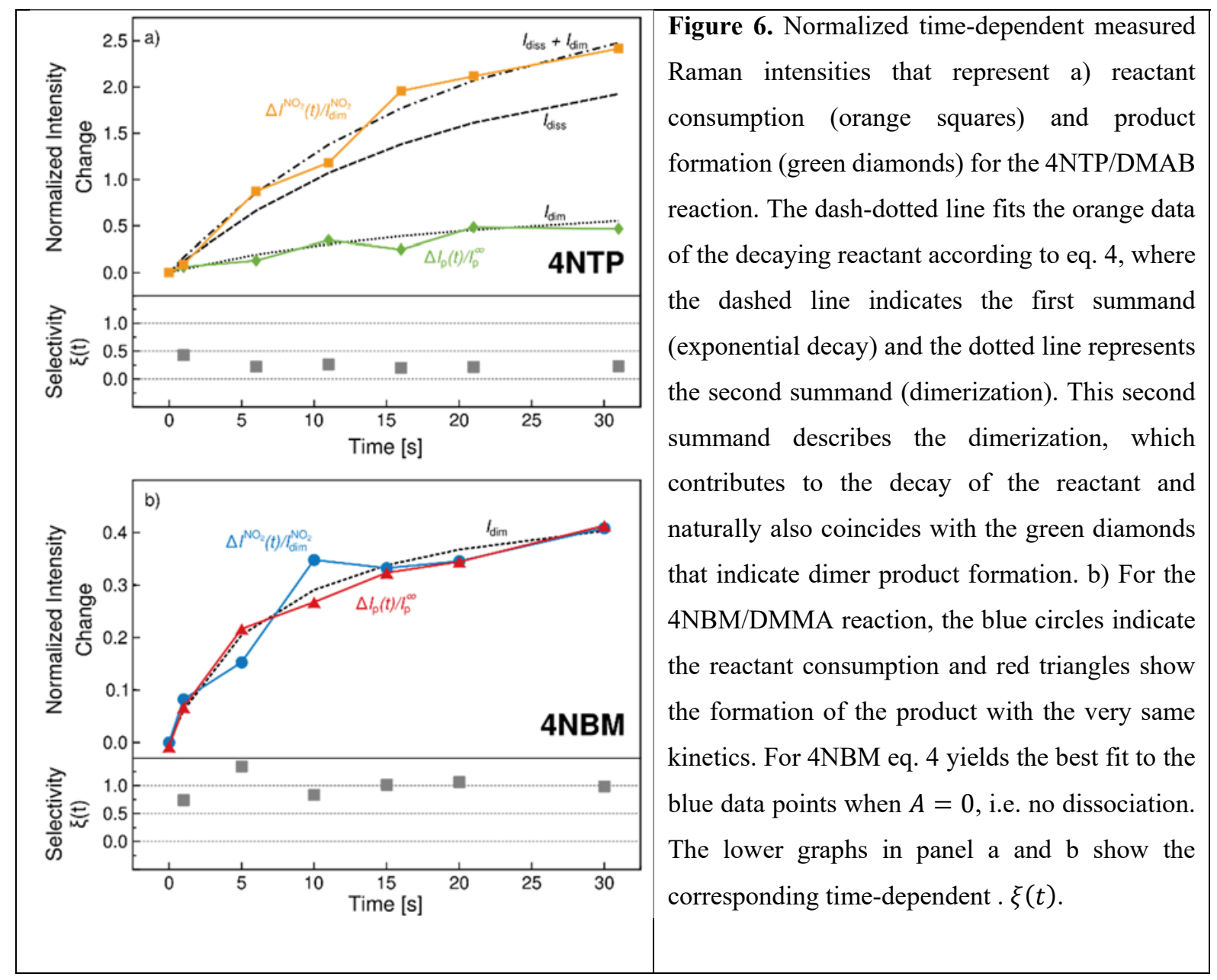

normalized intensity changes in Figure $6 \mathrm{~b}$ indicate that $40 \%$ of the $4-\mathrm{NBM}$ that dimerize according to our model have dimerized within 30 s. This is consistent with the $40 \%$ loss in intensity of the $\mathrm{NO}_{2}$ peak in Figure $3 \mathrm{~d}$. For the 4 NTP, Figure $6 \mathrm{a}$ also states that about $40 \%$ of the molecules that may dimerize have done that within 30s. However, a larger fraction of the molecules (180\%) undergo dissociation. This value is a consequence of the normalization with respect to $\mathrm{I}_{\mathrm{dim}}^{\mathrm{NO}_{2}}$ in our model. Essentially four times more 4NTP molecules undergo dissociation than dimerization in this time span, and a cross check with Figure 3c shows that an even larger fraction of 4NTP molecules does not react at all. Our model accounts for this fact, because eq. 3 describes the change of the Raman intensity $\Delta I^{\mathrm{NO}_{2}}(t)$.

From $I_{d i s}^{\mathrm{NO}_{2}}(t)$ and $I_{\text {dim }}^{\mathrm{NO}_{2}}(t)$ we can also calculate the time-dependent selectivity for dimerization by: 
(eq. 6)

$$
\xi(t) \equiv \frac{[P](t)}{\left[R_{0}\right]}=\frac{I_{d i m}^{\mathrm{NO}_{2}}(t)}{I_{d i m}^{N O_{2}}+I_{d i s}^{N O_{2}}}
$$

With a selectivity of about $100 \%$ for the dimerization pathway, exactly two reactant molecules are consumed to produce one dimer product molecule. The production transient of DMMA indeed corresponds to the consumption of 4NBM (Figure 6b). Hence the selectivity is approximately $\xi_{N B M}=$ 1 at all times. In contrast, the production of DMAB is much lower than the consumption of 4NTP (Figure 6a). The selectivity is initially around $\xi_{N T P}(t)=0.5$ and even decreases to $\xi_{N T P}(t)=0.25$ for later times. This clearly proves that not all consumed 4 NTP is converted to DMAB, but rather the majority of 4NTP molecules react to some other product or do not react at all.

\section{Discussion}

The XPS measurements show that the reduced work function from the gold surface to 4NBM is 600 meV larger compared to 4NTP, whereas the density of conduction band states is comparable for both systems. Consequently, charge transfer from the particles to 4NBM requires a much higher activation energy than charge transfer to 4NTP. This observation is supported by the computed electron affinities of the molecule-surface complex. On the other hand, the dimerization rate of 4NBM is much higher than of 4NTP. We suggest that the different reaction behavior originates from structural differences of the molecules and their concomitant motional freedom.

The activation energy of the dimerization step is determined by the energy needed for the combined structural rearrangement of the two involved reactant molecules. The flexibility of $4 \mathrm{NTP}$ is very limited, due to the strongly covalent S-Au bond involving the thiol group. Indeed, Sun and co-workers have recently shown that the dimerization of 4NTP and 4ATP only proceeds on ill-defined gold surfaces ${ }^{31}$, where the relative orientation of the reactants allowed the formation of DMAB with low intermolecular strain. The planar surfaces of the nanotriangles are very well defined. Hence, the DMAB formation 
preferentially occurs in nanogaps. The formation of the cis isomer requires higher energy. The free trans-DMAB molecule is about $0.7 \mathrm{eV}$ more stable than the corresponding cis isomer on the B3LYP/TZVP level of theory. On the other hand, Zhang and co-workers demonstrated that if the dimerization of plasmon-activated 4 NTP is prevented, e.g. if the intermolecular distance is too large, a dissociation channel of the nitro-group is activated that results in the production of $\mathrm{TP}^{39}$. We therefore conclude that the low selectivity factor $\xi_{N T P}(t)=0.25$ implies that only one out of four 4NTP molecules can find a suitably oriented reaction partner, while the other molecules convert to TP. However, as the Raman spectrum of TP largely coincides with the spectrum of 4NTP and has a much lower Raman cross section, we could not directly observe its formation.

Compared to 4NTP, the additional methylene group provides 4NBM with additional degrees of freedom to rearrange the orientation of the nitro group relative to the surface. This considerably lowers the activation energy for achieving the correct structural arrangement, which is reflected in the higher reaction rate for 4NBM compared to 4NTP. Moreover, it allows a higher fraction of 4NBM molecules to dimerize. The observed selectivity of $\xi_{N B M}(t)=1$ indeed suggests, that all activated 4 NBM molecules that undergo a reaction follow the dimerization pathway. However, this can be an exaggeration because molecules in plasmonic hot spots, which are located at the particle edges, contribute more to the signal.

Figure 1d schematically illustrates the various conformations that the 4-NBM molecule can take on a surface by rotation around the S-C bond, which are confirmed by the DFT modeling in Figure 4. Surely in a densely packed monolayer of species thiol-bonded to the Au surface will prohibit rotation because of steric hinderance. But it is well possible that the trans-isomer of DMMA can even be formed by adjacent molecules on the flat Au surface and thermal motion will allow the reactive group to assume favorable positions. 


\section{Conclusions}

In this article we investigated the origin of the kinetic limitation in plasmon-driven dimerization of nitro-benzenes adsorbed on gold nanoparticles. For this we compared the prototypical dimerization of 4-nitrothiophenol (4NTP) to 4,4'-dimercaptoazobenzene (DMAB) to the dimerization of the similar molecule 4-nitrobenzenemercaptane (4NBM) to 4,4'-dimercaptomethan-azobenzene (DMMA). A quantitative comparison of the dimerization kinetics shows that under the same reaction conditions both the rate and yield of the 4NBM to DMMA reaction is significantly higher than that of the 4NTP to DMAB. XPS measurements combined with DFT simulations rule out differences in the charge transfer as reason for this observation. In fact, the corresponding data suggest that the electron transfer from the surface to 4NTP is facilitated compared to 4NBM. On the other hand, the additional methylene group in 4NBM enhances the molecules' flexibility to assume an arrangement favorable for dimerization. We therefore conclude that the enhanced flexibility of the 4NBM molecule increases the probability for the dimerization step compared to 4NTP and hence causes a faster reaction kinetics. A proper molecular design thus allows tuning the selectivity of such reactions.

The experimental results confirm that the plasmon-driven dimerization of nitro-benzene compounds is limited in rate and yield by the dimerization rather than the electron transfer activation step of the reaction. It is therefore the unique capability of plasmonic reaction sites to provide both charges and thermal energy that allows the photo-driven $\mathrm{N}=\mathrm{N}-$ bond formation. In the design of future plasmonic nano-reactors, it must thus be carefully analyzed whether optimizing the generation of energetic electrons or nano-heating will foster the overall reaction. 


\section{Methods}

\section{Preparation of Gold Nanoparticles}

Citrate stabilized AuNPs with a diameter of $10 \pm 2 \mathrm{~nm}$ have been synthesized by reduction of $\mathrm{HAuCl}_{4}$ with trisodium citrate described elsewhere in detail. ${ }^{41}$ In order to exchange the ligands $450 \mathrm{ml}$ of AuNP solution have been washed with MilliQ filtered water using Amicon filters (15 ml, $30 \mathrm{kDa})$ at $3000 \mathrm{~g}$ for 10 minutes to reduce the citrate concentration in the solution and subsequently incubated overnight with $200 \mu \mathrm{M}$ 4-nitrothiophenol (4NTP) or 4-nitrobenzenemercaptan (4NBM), respectively. After two additional washing steps, the final volume has been adjusted to $150 \mathrm{ml}$.

AuNTs were prepared according to the recently described procedure, ${ }^{30}$ based on a one-step process in a mixed AOT/phospholipon vesicle phase. Poly $\left(\mathrm{N}, \mathrm{N}^{\prime}\right.$-diallyl-N, $\mathrm{N}^{\prime}$-dimethylammonium-alt-3,5-biscarboxyphenylmaleamic carboxylate (PalPhBisCarb), is used as a reducing and shape controlling agent. Briefly, $0.5 \mathrm{wt} \%$ phospholipid (PL90G; purity $>97 \%$ ) and $0.5 \mathrm{wt} \%$ dioctyl sodium sulfosuccinate (AOT) were mixed together in water together in the presence of 0.01 wt \% PalPhBisCarb. The dispersion was stirred for $24 \mathrm{~h}$ at room temperature. Afterwards, $2 \mathrm{mM}$ tetrachloroaurate precursor solution was added to the resulting template vesicle phase and heated up to $45{ }^{\circ} \mathrm{C}$ for $45 \mathrm{~min}$. The resulting purple colored-dispersion confirms the formation of the nanotriangles. The nanotriangles were then separated from spherical nanoparticles by a depletion flocculation step after adding a $0.02 \mathrm{M}$ AOT micelle solution. For the assembly of the NTs, injection of a mixture of ethanol-toluene (5:1) into the gold NTs solution forms a monolayer of the NTs at the liquid -air interface, which was then deposited on the silicon wafer after the solvent evaporation. The thiolated molecules (4NTP, 4NBM) were selfassembled on the NTs monolayer after immersing the NTs-functionalized wafers in an ethanolic solution of $5 \mathrm{mM}$ of these molecules for $6 \mathrm{~h}$. The wafers were washed with ethanol and water to remove the unattached molecules before the measurements. 


\section{XPS}

XPS measurements of isolated $\mathrm{Au}$ nanoparticles have been performed at the PLEIADES beamline at the synchrotron SOLEIL using the multipurpose source chamber (MPSC). ${ }^{42,43}$ The AuNPs have been brought to the gas-phase from a colloidal solution using TSI 3076 atomizer, with Argon as carrier gas. The solvent (water) has been removed by passing the aerosol through silica desiccators. The AuNPs enter the MPSC through a limiting orifice $(240 \mu \mathrm{m})$ and have been focused with a set of aerodynamic lenses. At the entrance of the VG Scienta R4000 hemispherical electron energy analyzer the AuNP beam has been crossed with the soft X-ray photon beam produced by a permanent magnet APPLE II type undulator, with a period of $80 \mathrm{~mm}$, in combination with a high-flux, $6001 / \mathrm{mm}$ grating of the modified Petersen plane grating monochromator used to monochromatize the synchrotron radiation. The XPS spectra were measured at the incident photon energies of $100 \mathrm{eV}$ for the valence band states and $400 \mathrm{eV}$ for the $\mathrm{Au}$ 4f states with an overall resolution of $157 \mathrm{meV}$ and $1089 \mathrm{meV}$, respectively . The binding energy of the $\mathrm{Au} 4 \mathrm{f}$ energy levels was calibrated according to the kinetic difference with respect to the $2 p$ and 3 s ionization edges of the carrier Argon gas $^{44}$, whereas for the valence states the $12.6 \mathrm{eV}$ line of gaseous water was used. ${ }^{45}$ The linearity of the kinetic energy scale has been validated by using $2 \mathrm{~s}, 2 \mathrm{p}, 3 \mathrm{p}$ and $3 \mathrm{~s}$ ionization edges ${ }^{44}$ and KLL Auger electrons ${ }^{46}$ of the carrier Argon gas, as discussed before. ${ }^{47}$ It should be noted that in the PLEIADES setup, the photoelectron lines from the focused AuNPs can be resolved from those produced by residual $\mathrm{H}_{2} \mathrm{O}$ solvent molecules and Ar carrier gas, which are not focused by the aerodynamic lens. ${ }^{47}$

\section{Raman Micro-Spectroscopy}

Raman spectra were recorded using a confocal Raman microscope (alpha 300; WITec, Ulm, Germany) coupled with a laser excitation of a wavelength at $785 \mathrm{~nm}$. The laser beam was focused through 10X (Nikon, NA=0.25) microscope objective, in order to average out sample inhomogeneities in the particle distribution. The spectra were acquired with a thermoelectrically cooled CCD detector (DU401A-BV, Andor, UK) placed behind the spectrometer (UHTS 300; WITec, Ulm, Germany). 


\section{Scanning Electron Microscopy}

The plasmonic substrates were imaged by scanning electron microscope (SEM) Supra55PV (ZEISS, Germany) operated at an acceleration voltage of $6 \mathrm{kV}$.

\section{DFT Calculations}

Full details of the calculations are described in the SI, sections S1 and S2. All calculations were performed with the quantum chemical package Gaussian $16^{48}$. Free molecules were optimized using DFT with the global hybrid functional B3LYP ${ }^{49,50}$ and the triple zeta valence polarized (TZVP) basis set $^{51}$. Normal mode analysis was performed to confirm the minimum nature of the optimized geometries and to compute Raman spectra. Computed harmonic frequencies were not scaled, and Raman signals were broadened by Lorentzians with a Full Width at Half Maximum of $15 \mathrm{~cm}^{-1}$. Molecules at surfaces were modelled using clusters with 1,2,10,20, and 30 gold atoms, representing up to three layers of an ideal $\mathrm{Au}(111)$ surface, with shortest $\mathrm{Au}-\mathrm{Au}$ distance of 2.88 Angstrom. Gold atoms were fixed in all optimizations and normal mode analyses including more than one gold atom. A bridge adsorption was assumed. Raman spectra were calculated for (one-layer) $\mathrm{Au}_{10}$ clusters (and the free molecules) only. For $\mathrm{Au}$, the LANL2TZ(f) ${ }^{52}$ effective core potential with the corresponding basis set (of triple zeta valence orbital quality and with $\mathrm{f}$ polarization functions) were used. Electron affinities were calculated by the Delta SCF method. For closed-shell singlet species, spin-restricted DFT was used, and unrestricted DFT (UB3LYP) for doublet ground states of, e.g., cluster-molecule complexes with an even number of gold atoms or negatively charged free molecules.

\section{Acknowledgements}

IB, RS and AM acknowledge support by the European Research Council (ERC; consolidator grant no. 772752), and we acknowledge beamtime at the synchrotron SOLEIL at the beamline PLEIADES through project 20191485 . 


\section{References}

(1) Mukherjee, S.; Zhou, L.; Goodman, A. M.; Large, N.; Ayala-Orozco, C.; Zhang, Y.; Nordlander, P.; Halas, N. J. Hot-electron-induced dissociation of $\mathrm{H} 2$ on gold nanoparticles supported on $\mathrm{SiO} 2$. Journal of the American Chemical Society 2014, 136, 64-67.

(2) Ren, X.; Tan, E.; Lang, X.; You, T.; Jiang, L.; Zhang, H.; Yin, P.; Guo, L. Observing reduction of 4-nitrobenzenthiol on gold nanoparticles in situ using surface-enhanced Raman spectroscopy.

Physical chemistry chemical physics : PCCP 2013, 15, 14196-14201.

(3) Shaik, F.; Peer, I.; Jain, P. K.; Amirav, L. Plasmon-Enhanced Multicarrier Photocatalysis. Nano letters 2018, 18, 4370-4376.

(4) Wilson, A. J.; Jain, P. K. Light-Induced Voltages in Catalysis by Plasmonic Nanostructures. Accounts of chemical research 2020, 53, 1773-1781.

(5) Yu, S.; Jain, P. K. Plasmonic photosynthesis of C1-C3 hydrocarbons from carbon dioxide assisted by an ionic liquid. Nature communications 2019, 10, 2022.

(6) Kim, Y.; Smith, J. G.; Jain, P. K. Harvesting multiple electron-hole pairs generated through plasmonic excitation of Au nanoparticles. Nature chemistry 2018, 10, 763-769.

(7) Koopman, W.; Sarhan, R. M.; Stete, F.; Schmitt, C. N. Z.; Bargheer, M. Decoding the kinetic limitations of plasmon catalysis: the case of 4-nitrothiophenol dimerization. Nanoscale [Online] 2020. (8) Xiao, Q.; Sarina, S.; Bo, A.; Jia, J.; Liu, H.; Arnold, D. P.; Huang, Y.; Wu, H.; Zhu, H. Visible Light-Driven Cross-Coupling Reactions at Lower Temperatures Using a Photocatalyst of Palladium and Gold Alloy Nanoparticles. ACS Catal. 2014, 4, 1725-1734.

(9) Liang, C.; Lu, Z.-A.; Wu, J.; Chen, M.-X.; Zhang, Y.; Zhang, B.; Gao, G.-L.; Li, S.; Xu, P. Recent Advances in Plasmon-Promoted Organic Transformations Using Silver-Based Catalysts. ACS applied materials \& interfaces 2020, 12, 54266-54284.

(10) Mohammadparast, F.; Dadgar, A. P.; Tirumala, R. T. A.; Mohammad, S.; Topal, C. O.; Kalkan, A. K.; Andiappan, M. C-C Coupling Reactions Catalyzed by Gold Nanoparticles: Evidence for Substrate-Mediated Leaching of Surface Atoms Using Localized Surface Plasmon Resonance Spectroscopy. J. Phys. Chem. C 2019, 123, 11539-11545.

(11) Sharma, K.; Kumar, M.; Bhalla, V. Aggregates of the pentacenequinone derivative as reactors for the preparation of $\mathrm{Ag} @ \mathrm{Cu} 2 \mathrm{O}$ core-shell NPs: an active photocatalyst for Suzuki and Suzuki type coupling reactions. Chemical communications (Cambridge, England) 2015, 51, 12529-12532.

(12) Sarhan, R. M.; Koopman, W.; Schuetz, R.; Schmid, T.; Liebig, F.; Koetz, J.; Bargheer, M. The importance of plasmonic heating for the plasmon-driven photodimerization of 4-nitrothiophenol. Scientific reports 2019, 9, 3060.

(13) Sarhan, R. M.; Koopman, W.; Pudell, J.-E.; Stete, F.; Rössle, M.; Herzog, M.; Schmitt, C. N. Z.; Liebig, F.; Koetz, J.; Bargheer, M. Scaling Up Nanoplasmon Catalysis: The Role of Heat Dissipation. J. Phys. Chem. C 2019, 123, 9352-9357.

(14) Yu, S.; Mohan, V.; Jain, P. K. Using plasmonically generated carriers as redox equivalents. MRS Bull. 2020, 45, 43-48.

(15) Zheng, B. Y.; Zhao, H.; Manjavacas, A.; McClain, M.; Nordlander, P.; Halas, N. J.

Distinguishing between plasmon-induced and photoexcited carriers in a device geometry. Nature communications 2015, 6, 7797.

(16) Qiu, J.; Wei, W. D. Surface Plasmon-Mediated Photothermal Chemistry. J. Phys. Chem. C 2014, $118,20735-20749$.

(17) Jain, P. K. Taking the Heat Off of Plasmonic Chemistry. J. Phys. Chem. C 2019, 123, 2434724351.

(18) Mukherjee, S.; Libisch, F.; Large, N.; Neumann, O.; Brown, L. V.; Cheng, J.; Lassiter, J. B.; Carter, E. A.; Nordlander, P.; Halas, N. J. Hot electrons do the impossible: plasmon-induced dissociation of $\mathrm{H} 2$ on Au. Nano letters 2013, 13, 240-247. 
(19) Seemala, B.; Therrien, A. J.; Lou, M.; Li, K.; Finzel, J. P.; Qi, J.; Nordlander, P.; Christopher, P. Plasmon-Mediated Catalytic O2 Dissociation on Ag Nanostructures: Hot Electrons or Near Fields? ACS Energy Lett. [Online] 2019, 1803-1809.

(20) Dong, B.; Fang, Y.; Chen, X.; Xu, H.; Sun, M. Substrate-, wavelength-, and time-dependent plasmon-assisted surface catalysis reaction of 4-nitrobenzenethiol dimerizing to $\mathrm{p}, \mathrm{p}$ 'dimercaptoazobenzene on $\mathrm{Au}, \mathrm{Ag}$, and $\mathrm{Cu}$ films. Langmuir : the ACS journal of surfaces and colloids 2011, 27, 10677-10682.

(21) Dong, B.; Fang, Y.; Xia, L.; Xu, H.; Sun, M. Is 4-nitrobenzenethiol converted to p,p'dimercaptoazobenzene or 4-aminothiophenol by surface photochemistry reaction? J. Raman Spectrosc. 2011, 42, 1205-1206.

(22) Kim, K.; Choi, J.-Y.; Shin, K. S. Surface-Enhanced Raman Scattering of 4-Nitrobenzenethiol and 4-Aminobenzenethiol on Silver in Icy Environments at Liquid Nitrogen Temperature. J. Phys. Chem. C 2014, 118, 11397-11403.

(23) Seah, M. P.; Gilmore, I. S.; Beamson, G. XPS: binding energy calibration of electron spectrometers 5?re-evaluation of the reference energies. Surf. Interface Anal. 1998, 26, 642-649.

(24) Powell, C. J.; Jablonski, A. Evaluation of Calculated and Measured Electron Inelastic Mean Free Paths Near Solid Surfaces. Journal of Physical and Chemical Reference Data 1999, 28, 19-62.

(25) Vericat, C.; Vela, M. E.; Benitez, G.; Carro, P.; Salvarezza, R. C. Self-assembled monolayers of thiols and dithiols on gold: new challenges for a well-known system. Chemical Society reviews 2010, $39,1805-1834$.

(26) Ford, W. E.; Gao, D.; Knorr, N.; Wirtz, R.; Scholz, F.; Karipidou, Z.; Ogasawara, K.; Rosselli, S.; Rodin, V.; Nelles, G.; Wrochem, F. von. Organic dipole layers for ultralow work function electrodes. ACS nano 2014, 8, 9173-9180.

(27) Zojer, E.; Taucher, T. C.; Hofmann, O. T. The Impact of Dipolar Layers on the Electronic Properties of Organic/Inorganic Hybrid Interfaces. Adv. Mater. Interfaces 2019, 6, 1900581.

(28) Cahen, D.; Kahn, A. Electron Energetics at Surfaces and Interfaces: Concepts and Experiments. Adv. Mater. 2003, 15, 271-277.

(29) Lindstrom, C. D.; Zhu, X.-Y. Photoinduced electron transfer at molecule-metal interfaces. Chemical reviews 2006, 106, 4281-4300.

(30) Liebig, F.; Sarhan, R. M.; Sander, M.; Koopman, W.; Schuetz, R.; Bargheer, M.; Koetz, J. Deposition of Gold Nanotriangles in Large Scale Close-Packed Monolayers for X-ray-Based Temperature Calibration and SERS Monitoring of Plasmon-Driven Catalytic Reactions. ACS applied materials \& interfaces 2017, 9, 20247-20253.

(31) Sun, J.-J.; Su, H.-S.; Yue, H.-L.; Huang, S.-C.; Huang, T.-X.; Hu, S.; Sartin, M. M.; Cheng, J.; Ren, B. Role of Adsorption Orientation in Surface Plasmon-Driven Coupling Reactions Studied by Tip-Enhanced Raman Spectroscopy. The journal of physical chemistry letters 2019, 10, 2306-2312. (32) Rajalingam, K.; Hallmann, L.; Strunskus, T.; Bashir, A.; Wöll, C.; Tuczek, F. Self-assembled monolayers of benzylmercaptan and para-cyanobenzylmercaptan on gold: surface infrared spectroscopic characterization. Physical chemistry chemical physics : PCCP 2010, 12, 4390-4399. (33) Nishi, N.; Hobara, D.; Yamamoto, M.; Kakiuchi, T. Orientation of o -, m -, and p Methylbenzylmercaptans Adsorbed on $\mathrm{Au}(111)$ Probed by Broad-Bandwidth Sum Frequency Generation Spectroscopy. Langmuir 2003, 19, 6187-6192.

(34) Tao, Y.-T.; Wu, C.-C.; Eu, J.-Y.; Lin, W.-L.; Wu, K.-C.; Chen, C. Structure Evolution of Aromatic-Derivatized Thiol Monolayers on Evaporated Gold. Langmuir 1997, 13, 4018-4023. (35) Abdelsalam, M. Surface enhanced raman scattering of aromatic thiols adsorbed on nanostructured gold surfaces. Open Chemistry 2009, 7, 446-453.

(36) Tang, X.; Cai, W.; Yang, L.; Liu, J. Monitoring plasmon-driven surface catalyzed reactions in situ using time-dependent surface-enhanced Raman spectroscopy on single particles of hierarchical peony-like silver microflowers. Nanoscale 2014, 6, 8612-8616. 
(37) van Schrojenstein Lantman, E. M.; Gijzeman, O. L. J.; Mank, A. J. G.; Weckhuysen, B. M. Investigation of the Kinetics of a Surface Photocatalytic Reaction in Two Dimensions with Surfaceenhanced Raman Scattering. ChemCatChem 2014, 6, 3342-3346.

(38) Choi, H.-K.; Park, W.-H.; Park, C.-G.; Shin, H.-H.; Lee, K. S.; Kim, Z. H. Metal-Catalyzed Chemical Reaction of Single Molecules Directly Probed by Vibrational Spectroscopy. Journal of the American Chemical Society 2016, 138, 4673-4684.

(39) Zhang, Z.; Deckert-Gaudig, T.; Singh, P.; Deckert, V. Single molecule level plasmonic catalysis - a dilution study of p-nitrothiophenol on gold dimers. Chemical communications (Cambridge,

England) 2015, 51, 3069-3072.

(40) Schürmann, R.; Luxford, T. F. M.; Vinklárek, I. S.; Kočišek, J.; Zawadzki, M.; Bald, I. Interaction of 4-nitrothiophenol with low energy electrons: Implications for plasmon mediated reactions. The Journal of Chemical Physics 2020, 153, 104303.

(41) Schürmann, R.; Ebel, K.; Nicolas, C.; Milosavljević, A. R.; Bald, I. Role of Valence Band States and Plasmonic Enhancement in Electron-Transfer-Induced Transformation of Nitrothiophenol. The journal of physical chemistry letters 2019, 10, 3153-3158.

(42) Sublemontier, O.; Nicolas, C.; Aureau, D.; Patanen, M.; Kintz, H.; Liu, X.; Gaveau, M.-A.; Le Garrec, J.-L.; Robert, E.; Barreda, F.-A.; Etcheberry, A.; Reynaud, C.; Mitchell, J. B.; Miron, C. Xray Photoelectron Spectroscopy of Isolated Nanoparticles. The journal of physical chemistry letters 2014, 5, 3399-3403.

(43) Lindblad, A.; Söderström, J.; Nicolas, C.; Robert, E.; Miron, C. A multi purpose source chamber at the PLEIADES beamline at SOLEIL for spectroscopic studies of isolated species: cold molecules, clusters, and nanoparticles. The Review of scientific instruments 2013, 84, 113105.

(44) Thompson, A. C.; Attwood, D. T.; Gullikson, E. M.; Howells, M. R.; Kortright, J. B.; Robinson, A. L.; Underwood, J. H.; Kim, K.-J.; Kirz, J.; Lindau, I.; Pianetta, P.; Winick, H.; Williams, G. P.; Scofield, J. H. X-RAY DATA BOOKLET: Berkeley, 2009.

(45) Reutt, J. E.; Wang, L. S.; Lee, Y. T.; Shirley, D. A. Molecular beam photoelectron spectroscopy and femtosecond intramolecular dynamics of $\mathrm{H} 2 \mathrm{O}+$ and D $2 \mathrm{O}+$. The Journal of Chemical Physics 1986, 85, 6928-6939.

(46) Werme, L. O.; Bergmark, T.; Siegbahn, K. The L2,3MM Auger Spectrum of Argon. Phys. Scr. 1973, 8, 149-153.

(47) Danilović, D.; Božanić, D. K.; Dojčilović, R.; Vukmirović, N.; Sapkota, P.; Vukašinović, I.; Djoković, V.; Bozek, J.; Nicolas, C.; Ptasinska, S.; Milosavljević, A. R. Aerosol Synthesis and GasPhase Photoelectron Spectroscopy of Ag-Bi-I Nanosystems. J. Phys. Chem. C 2020, 124, 2393023937.

(48) Frisch, M. J.; Trucks, G. W.; Schlegel, H. B.; Scuseria, G. E.; Robb, M. A.; Cheeseman, J. R.; Scalmani, G.; Barone, V.; Petersson, G. A.; Nakatsuji, H.; Li, X.; Caricato, M.; Marenich, A. V.; Bloino, J.; Janesko, B. G.; Gomperts, R.; Mennucci, B.; Hratchian, H. P.; Ortiz, J. V.; Izmaylov, A. F.; Sonnenberg, J. L.; Williams-Young, D.; Ding, F.; Lipparini, F.; Egidi, F.; Goings, J.; Peng, B.; Petrone, A.; Henderson, T.; Ranasinghe, D.; Zakrzewski, V. G.; Gao, J.; Rega, N.; Zheng, G.; Liang, W.; Hada, M.; Ehara, M.; Toyota, K.; Fukuda, R.; Hasegawa, J.; Ishida, M.; Nakajima, T.; Honda, Y.; Kitao, O.; Nakai, H.; Vreven, T.; Throssell, K.; Montgomery, J. A., Jr.; Peralta, J. E.; Ogliaro, F.; Bearpark, M. J.; Heyd, J. J.; Brothers, E. N.; Kudin, K. N.; Staroverov, V. N.; Keith, T. A.; Kobayashi, R.; Normand, J.; Raghavachari, K.; Rendell, A. P.; Burant, J. C.; Iyengar, S. S.; Tomasi, J.; Cossi, M.; Millam, J. M.; Klene, M.; Adamo, C.; Cammi, R.; Ochterski, J. W.; Martin, R. L.; Morokuma, K.; Farkas, O.; Foresman, J. B.; Fox, D. J. Gaussian16, Revision C.01; Gaussian Inc., Wallingford CT, 2016.

(49) Becke, A. D. Density-functional thermochemistry. III. The role of exact exchange. The Journal of Chemical Physics 1993, 98, 5648-5652. 
(50) Stephens, P. J.; Devlin, F. J.; Chabalowski, C. F.; Frisch, M. J. Ab Initio Calculation of Vibrational Absorption and Circular Dichroism Spectra Using Density Functional Force Fields. $J$. Phys. Chem. 1994, 98, 11623-11627.

(51) Schäfer, A.; Huber, C.; Ahlrichs, R. Fully optimized contracted Gaussian basis sets of triple zeta valence quality for atoms Li to Kr. The Journal of Chemical Physics 1994, 100, 5829-5835.

(52) Roy, L. E.; Hay, P. J.; Martin, R. L. Revised Basis Sets for the LANL Effective Core Potentials. Journal of chemical theory and computation 2008, 4, 1029-1031. 